\title{
Airway management in inhalation injury: a case series
}

\author{
Suneel Ramesh Desai ${ }^{1,2}$, MBChB, FRCA, Delong Zeng ${ }^{3}$, MBBS, Si Jack Chong $^{3}$, MMed, FAMS
}

\begin{abstract}
Inhalation injury is a serious consequence of a fire or an explosion, with potential airway compromise and respiratory complications. We present a case series of five patients with inhalational burns who presented to Singapore General Hospital and discuss our approach to their early management, including early evaluation and planning for the upper and lower airway, coexisting cutaneous burns, and monitoring their ICU (intensive care unit) severity of illness, sepsis and acute respiratory distress syndrome. All five patients suffered various grades of inhalation injury. The patients were initially assessed by nasolaryngoscopy, and three patients were prophylactically intubated before being sent to the emergency operating theatre for definitive airway and burns management with fibreoptic bronchoscopy. All patients were successfully extubated and discharged stable. Various complications can arise as a result of an inhalation injury. Based on our cases and literature review, we propose a standardised workflow for patients with inhalation injury.
\end{abstract}

Keywords: airway management, bronchoscopy, burns, inhalation injury, ventilation

\section{INTRODUCTION}

Inhalation injuries, which may occur as a result of a fire or an explosion in an enclosed space, can be associated with cutaneous facial burns and coexisting trauma. Specific complications include thermal injury and swelling of the upper and lower airway, bronchospasm, bronchial obstruction with carbonaceous sputum plugs, pneumonia, acute respiratory distress syndrome (ARDS), carbon monoxide toxicity and cyanide toxicity, as well as the complications of coexisting cutaneous burns and trauma, if present. Hence, the initial triage and approach to patient management focuses on these areas. We present a case series of five patients with inhalational burns in 2017 (Table I), all of whom required endotracheal intubation and mechanical ventilation in the burns intensive care unit (BICU). We herein discuss our approach to these patients' early management, including early evaluation and planning for ongoing management of: (a) the upper airway; (b) lower airway; (c) coexisting cutaneous burns, trauma and medical conditions; and (d) monitoring the intensive care unit (ICU) severity of illness, sepsis and ARDS. Patient outcomes are summarised in Table II.

This retrospective review of the burns database was approved by the SingHealth Centralised Institutional Review Board. Written informed consent was obtained from the patients for publication of this case report and the accompanying images. Data was collected from the internal network database of Singapore General Hospital, Singapore.

\section{CASE SERIES}

\section{Patient 1}

A 63-year-old man had fallen asleep when his candle dropped on the living room floor and set fire to the windowsill. He suffered an inhalation injury but no cutaneous burns. At presentation to the accident and emergency department (A\&E), the patient was well with no stridor or change of voice. On bedside nasoendoscopy, he was found to have minimal soot in the anterior and posterior nasal space, with some soot in the glottis, base of the tongue, posterior cricoid and subglottic region. The larynx was not swollen.

Although chest radiography was clear on admission, the patient desaturated 24 hours after presentation. Wheeze and transmitted sounds were heard on auscultation. Subsequent chest radiography demonstrated bilateral lower lobe collapse with increased pulmonary congestion and consolidation, suggestive of pneumonia. Arterial blood gas showed Type 1 respiratory failure and metabolic acidosis. The patient was transferred emergently to the emergency operating theatre (EOT) for rapid sequence induction, endotracheal intubation and initiation of mechanical ventilation, and subsequently transferred to the BICU. Bronchoscopy showed a significant amount of thick mucous plugging, leading to pneumonia. In view of the thick mucous plugging, this was considered a Grade 3 inhalation injury based on the Endorf and Gamelli classification.

At the BICU, regular aggressive suctioning was done and bronchoalveolar lavage was performed. Daily arterial blood gas was monitored and serial chest radiographs were obtained. Intravenous piperacillin-tazobactam was given for pneumonia and intravenous vancomycin for coagulase-negative staphylococci on blood cultures. The patient was ventilated for five days and remained in the BICU for eight days. The pneumonia resolved while he was in the BICU, as shown by serial chest radiography. The patient was stable throughout his stay in the BICU and remained so until discharge.

\section{Patient 2}

A 32-year-old man was in a parking lot trying to jump-start a car battery when the engine exploded. He suffered 18\% total body surface area (TBSA) burns as well as inhalation injury. At initial presentation at the $\mathrm{A} \& \mathrm{E}$, the patient was alert but disorientated and moaning in pain. He was in an altered mental state with a Glasgow

${ }^{1}$ Department of Surgical Intensive Care, ${ }^{2}$ Department of Anaesthesiology, ${ }^{3}$ Department of Burns and Plastic Surgery, Singapore General Hospital, Singapore Correspondence: Dr Delong Zeng, Medical Officer, Department of Burns and Plastic Surgery, Singapore General Hospital, Outram Road, Singapore 169608. delong.zeng@mohh.com.sg 
Table I. Patient characteristics.

\begin{tabular}{|c|c|c|c|c|c|}
\hline Details & Case 1 & Case 2 & Case 3 & Case 4 & Case 5 \\
\hline Age (yr) & 63 & 32 & 47 & 23 & 28 \\
\hline Gender & Male & Male & Male & Male & Male \\
\hline $\mathrm{BMI}\left(\mathrm{kg} / \mathrm{m}^{2}\right)$ & 22.8 & 23.9 & 23.2 & 26.5 & 23.8 \\
\hline GCS score on arrival at A\&E & 15 & 14 & 15 & 13 & 15 \\
\hline Intubation location (RSI) & EOT & $A \& E$ & A\&E & $A \& E$ & EOT \\
\hline TBSA cutaneous burns (\%) & No burns & 18 & 18 & 22.5 & 20.5 \\
\hline Burns depth & NA & Partial thickness & Partial thickness & Partial thickness & Partial thickness \\
\hline Facial burns & No & Yes & Yes & Yes & Yes \\
\hline
\end{tabular}

A\&E: accident and emergency department; BMI: body mass index; EOT: emergency operating theatre; GCS: Glasgow Coma Scale; NA: not applicable; RSI: rapid sequence induction; TBSA: total body surface area

Table II. Patient outcomes.

\begin{tabular}{|c|c|c|c|c|c|}
\hline Details & Case 1 & Case 2 & Case 3 & Case 4 & Case 5 \\
\hline History of inhalation injury & Yes & Yes & Yes & Yes & Yes \\
\hline Inhalation injury* & Yes & Yes & Yes & Yes & Yes \\
\hline Bronchoscopy score & 3 & 2 & 2 & 2 & 1 \\
\hline APACHE II score & 21 & 8 & 9 & 14 & 14 \\
\hline SOFA score & 11 & 5 & 3 & 1 & 4 \\
\hline ARDS $^{+}$ & Severe & No & No & No & Mild \\
\hline Pneumonia & Yes & No & No & No & No \\
\hline Acute kidney injury ${ }^{\ddagger}$ & Yes & No & No & No & No \\
\hline Ventilator duration (day) & 5 & 2 & 3 & 2 & 3 \\
\hline ICU LOS (day) & 8 & 2 & 3 & 3 & 3 \\
\hline Hospital LOS (day) & 24 & 10 & 12 & 16 & 23 \\
\hline Mortality & No & No & No & No & No \\
\hline
\end{tabular}

*Positive nasolaryngoscopy findings for inhalation injury. †Based on Berlin criteria. ¥Based on Kidney Disease: Improving Global Outcomes guidelines APACHE: Acute Physiology And Chronic Health Evaluation; ARDS: acute respiratory distress syndrome; ICU: intensive care unit; LOS: length of stay; SOFA: sequential organ failure assessment

Coma Scale (GCS) score of 14/15. Bedside nasoendoscopy showed that the upper respiratory tract was swollen and the vocal cords mobile but slightly oedematous. No carbonaceous sputum was observed in the airways. The patient was intubated prophylactically at the A\&E and sent to the EOT for a burns scrubdown and Biobrane ${ }^{\mathrm{TM}}$ dressing (Smith \& Nephew, Singapore) application. Bronchoscopy was performed prior to surgery. The lower airway was normal with no swelling or particulate matter; however, erythema was present throughout. There was upper airway swelling. Grade 2 inhalation injury was identified.

In the BICU, the patient remained stable. Arterial blood gas was consistent with his condition and vitals were non-significant. The patient remained stable throughout his stay.

\section{Patient 3}

The patient, a 47-year-old taxi driver, had stepped out of his taxi when the car engine exploded. A second explosion caused the injury. He had 18\% TBSA burns and inhalation injury. At initial presentation, the patient was alert, orientated and spoke clearly. There was no hoarseness of voice or stridor, and oedematous upper airway was seen on nasoendoscopy. There was no soot.

The patient was intubated prophylactically in the A\&E in view of the laryngoscopy findings, although he was clinically stable.
He was subsequently sent to the EOT for a burns scrubdown and Biobrane dressing application. Bronchoscopy was performed at the end of the operation. There was erythema, friable mucosa and bronchorrhoea but no significant carbonaceous plugs. However, the patient had mucosal oedema in the trachea and the main airways, with complete occlusion of the right middle lobe of the lung and significant intrabronchial haemorrhage, especially in the left lower lobe. This was classified as a Grade 2 inhalation injury.

The patient was noted to have some blood-stained aspirates postoperatively in the BICU. Oesophagogastroduodenoscopy showed mild non-erosive gastritis. He remained stable in the BICU otherwise and was successfully extubated.

\section{Patient 4}

A 23-year-old man was working in the engine room on a cargo ship when the main engine exploded and set the room on fire. He suffered $22.5 \%$ TBSA cutaneous burns and inhalation injury. At the time of presentation, the patient was able to obey commands and open his eyes spontaneously. However, he was not able to answer any questions and could only mumble, with a GCS score of 13/15. No hoarseness of voice or stridor was heard. Nasoendoscopy showed soot in the nasal aperture and erythema throughout the airway but no oedema. 
In view of the potential for worsening airway oedema, the patient was prophylactically intubated in the A\&E by direct laryngoscopy even though he was able to speak. He was transferred to the operating room urgently for a burns scrubdown and Biobrane dressing application. Fibreoptic bronchoscopy at the end of the procedure identified a Grade 2 inhalation injury with a moderate amount of erythema at the carina and the left main bronchus, and mild erythema and bronchorrhoea in the right main bronchus. Distal to the main bronchi bilaterally, the lower bronchial tree was free from erythema or carbonaceous deposits. Bronchoalveolar lavage was performed with $20 \mathrm{~mL}$ normal saline. The patient was transferred to the BICU and was sedated and ventilated following the procedure. He remained stable in the BICU.

\section{Patient 5}

A 28-year-old man was involved in the same incident as Patient 4 , an engine room explosion aboard a cargo ship. He suffered 20.5\% TBSA burns and inhalation injury. On initial presentation at the A\&E, nasoendoscopy was performed. There was no soot, but mild erythema and oedema was present along the upper airway.

The patient was transferred emergently to the EOT to undergo a burns scrubdown and Biobrane dressing application. Fibreoptic bronchoscopy at the end of the procedure revealed a Grade 1 inhalation injury with patchy areas of erythematous lower airway mucosa. There were minimal carbonaceous deposits and some bronchorrhoea. Bronchoalveolar lavage with $40 \mathrm{~mL}$ normal saline was performed. The patient was again taken to the operating theatre two days after Biobrane dressing application for wound inspection and skin grafting for his deep dermal burns. He was initially agitated and required additional sedation but otherwise remained stable in the BICU.

\section{DISCUSSION}

We herein discuss the grading of the severity of inhalation injury, based on clinical history, examination findings, and upper and lower airway endoscopy findings. In addition, we discuss our approach to airway management and extubation assessment based on a review of the existing literature and our case series.

Initial evaluation of a patient presenting with a history of inhalation injury includes the standard Advanced Trauma Life Support (ATLS) approach. ${ }^{(1)}$ This includes a history of the mechanism of injury, alerts, allergies and comorbidities, and a complete primary and secondary survey. Immediate assessment includes evaluation of conscious level and vital signs, airway with cervical spine stabilisation, breathing, circulation, and coexisting whole body burns and trauma.

Inhalation injury is typically associated with burns to the face, neck and chest, as well as a large percentage of TBSA burned. However, it may occur even in the absence of cutaneous burns. A history of a fire or explosion within a closed space, or direct injury to the face with steam, chemicals or fire, suggest a high likelihood of inhalation injury. The presence of carbonaceous debris in the mouth or nose and singeing of the nasal hairs are particularly suggestive of inhalation injury. Other clinical features may include facial or upper airway swelling, hoarse voice, drooling of saliva, dysphagia and stridor. ${ }^{(2)}$

Lower airway inflammation may lead to ARDS with noncardiogenic pulmonary oedema related to inflammatory capillary leak, as well as bronchospasm, bronchorrhoea and impaired sputum clearance. The precipitation of carbonaceous soot particles in the lower tracheobronchial tree predisposes one to bronchial obstruction, lobar collapse and pneumonia. Other possible respiratory complications include carbon monoxide or cyanide toxicity, pneumothorax from blast injury, pulmonary aspiration related to reduced conscious level, chest wall restriction from circumferential burns around the thorax, and atelectasis related to abdominal distension. ${ }^{(3)}$

The immediate aims of management are summarised as follows: ${ }^{(4,5)}$

- Administer high-flow face mask oxygen via a nonrebreathing face mask to all patients in view of the risk of carbon monoxide toxicity.

- $\quad$ Complete ATLS primary survey, then the secondary survey when the primary survey has been completed, vital signs are normalising and resuscitation efforts have been established.

- Perform upper airway fibreoptic nasolaryngoscopy under local anaesthesia to evaluate for: carbonaceous debris in the pharynx or larynx; and erythema or swelling of the pharynx, aryepiglottic folds and vocal cords.

- Limit intravenous fluid therapy prior to definitive airway management in view of the risk of airway swelling and ARDS.

- Transfer the patient emergently to the operating room for definitive airway management and lung-protective ventilation; diagnostic fibreoptic bronchoscopy to confirm the diagnosis of lower airway inhalation injury, with bronchoalveolar lavage if needed to clear any obstructing carbonaceous plugs; and damage control surgery (escharotomy, $<5 \%$ excision and debridement of necrotic areas, and Biobrane application).

Several studies have identified factors associated with the need for early endotracheal intubation. ${ }^{(6-8)}$ These include soot in the oral cavity; facial and neck burns; high TBSA of cutaneous burns $>27 \%$; use of accessory respiratory muscles; elevated carboxyhaemoglobin (COHb) levels; and fibreoptic laryngoscopy findings of oedema, erythema or carbonaceous deposits on the true or false vocal cords. Importantly, features such as stridor, hoarse voice, drooling, dysphagia and singed nasal hair do not appear to be predictive of the need for early intubation, whereas fibreoptic laryngoscopy has emerged as a useful triage tool, with a high positive and negative predictive value. ${ }^{(9)}$ Goh et al ${ }^{(10)}$ described a modified triage system used in 22 patients presenting to the A\&E following a smoke inhalation mass casualty incident. Clinical history, symptoms, signs and respiratory risk factors such as asthma were used for preliminary screening. Patients with risk factors for inhalation injury underwent fibreoptic laryngoscopy, chest radiography and arterial blood sampling for analysis of arterial blood gases and $\mathrm{COHb}$ levels. Patients with upper airway erythema or elevated $\mathrm{COHb}$ levels were admitted to hospital, whereas all other patients could be discharged home. 


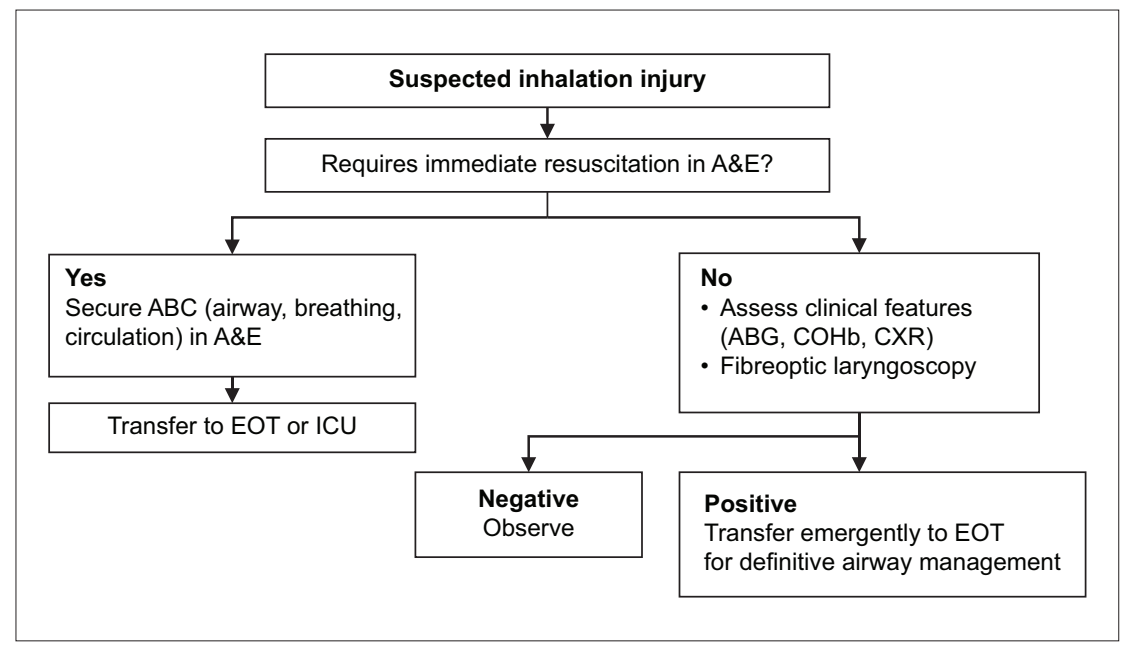

Fig. 1 Flowchart shows our institution's protocol on intubating a patient with suspected inhalation injury. A\&E: accident and emergency department; ABG: arterial blood gas; $\mathrm{COHb}$ : carboxyhaemoglobin; CXR: chest radiography; EOT: emergency operating theatre; ICU: intensive care unit

Ikonomidis et al ${ }^{(11)}$ conducted a prospective observational study in 100 patients with inhalation injury, in whom an endoscopic airway assessment was performed in the first few hours. An upper and lower airway endoscopy score was described, and lesions were classified as confined to the upper airway or with tracheobronchial extension. This is summarised in Box 1.

However, while upper airway fibreoptic laryngoscopy is well tolerated and highly predictive of the need for intubation, it may be dangerous to attempt lower airway bronchoscopy in an unintubated spontaneously breathing patient with inhalation injury in the $\mathrm{A} \& \mathrm{E}$, as required in the Ikonomidis classification system. In addition, lower airway bronchoscopy has a different purpose from upper airway laryngoscopy in inhalation injury. Whereas upper airway laryngoscopy is useful to predict the need for intubation, lower airway bronchoscopy is mainly useful in predicting the duration of mechanical ventilation, extubation planning, sputum clearance strategy and diagnosis of pneumonia.

In our practice, we adopted a simple binary (yes/no) classification of upper airway laryngoscopy findings similar to that used by Muehlberger et $\mathrm{al}^{\left({ }^{(9)}\right.}$ and Goh et al, ${ }^{(10)}$ compared with the more detailed classification proposed by Ikonomidis et al. ${ }^{(11)}$ Hence, a stable patient with suspected inhalation injury receives an upper airway fibreoptic laryngoscopy, chest radiography, and arterial blood sampling for arterial blood gases and $\mathrm{CoHb}$ levels in the $\mathrm{A} \& \mathrm{E}$, in addition to a standard clinical assessment. If any of these investigations or clinical features is abnormal, the patient is immediately transferred to the operating theatre for definitive airway management (Fig. 1). Fibreoptic bronchoscopy evaluation of the lower airway is only performed after the airway is secured, ventilation controlled and primary damage control surgery for coexisting cutaneous burns or other injuries has been completed.

In the presence of inhalation injury, further airway swelling is likely once intravenous fluid resuscitation has begun, hence it is essential that the upper airway is secured and ventilation controlled urgently even if the patient has no respiratory failure and is fully conscious at the time of presentation. In patients presenting to the $A \& E$ in severe shock, endotracheal intubation may be required as part of initial resuscitation, particularly in the context
Box 1. Ikonomidis et al's ${ }^{(11)}$ classification of endoscopic grade in inhalation injury:

Grade 1: Oedema, hyperaemia, hypersecretion

Grade 2: Bullous mucosal detachment, erosion, exudates

Grade 3: Profound ulcers, necrosis

of a reduced consciousness level, impending cardiorespiratory arrest, refractory hypoxaemia or refractory hypotension. Such patients may be too unstable to transfer to the operating room without first securing the airway. Coexisting cyanide toxicity, shock, and hypoxic or traumatic brain injury should be suspected in such cases. However, in most cases, patients with the following indications are stable enough to be transferred emergently for a planned endotracheal intubation in the operating room: ${ }^{(12,13)}$ positive upper airway nasolaryngoscopy and planned fibreoptic bronchoscopy; reduced consciousness level; respiratory failure; stridor; facial or neck burns, singed nasal hair, or soot around the nares or mouth; facial or oropharyngeal swelling; inhalation injury; circumferential chest burns restricting breathing; planned damage control surgery for burns debridement and coverage; and planned interhospital transfers to specialist burns centres.

All patients with inhalation injury, burns, trauma or any critical illness are anticipated to have a risk of difficult endotracheal intubation, difficult mask ventilation, pulmonary aspiration and cervical spine instability if there is coexisting trauma. In addition, respiratory and haemodynamic instability is anticipated following intubation and initiation of mechanical ventilation. In inhalation injury, the airway is dynamically evolving with increasing facial and airway swelling over time, particularly after the initiation of intravenous fluid therapy. This makes failed intubation, potentially resulting in hypoxic cardiac arrest and/or hypoxic ischaemic encephalopathy, more likely with any delay. Hence, early transfer to the EOT for definitive airway management is considered a time-critical intervention in patients with inhalation injury, as specialised airway equipment and staff, including anaesthesiologists and surgeons, would be available to perform a surgical airway or local anaesthetic tracheostomy if required. 


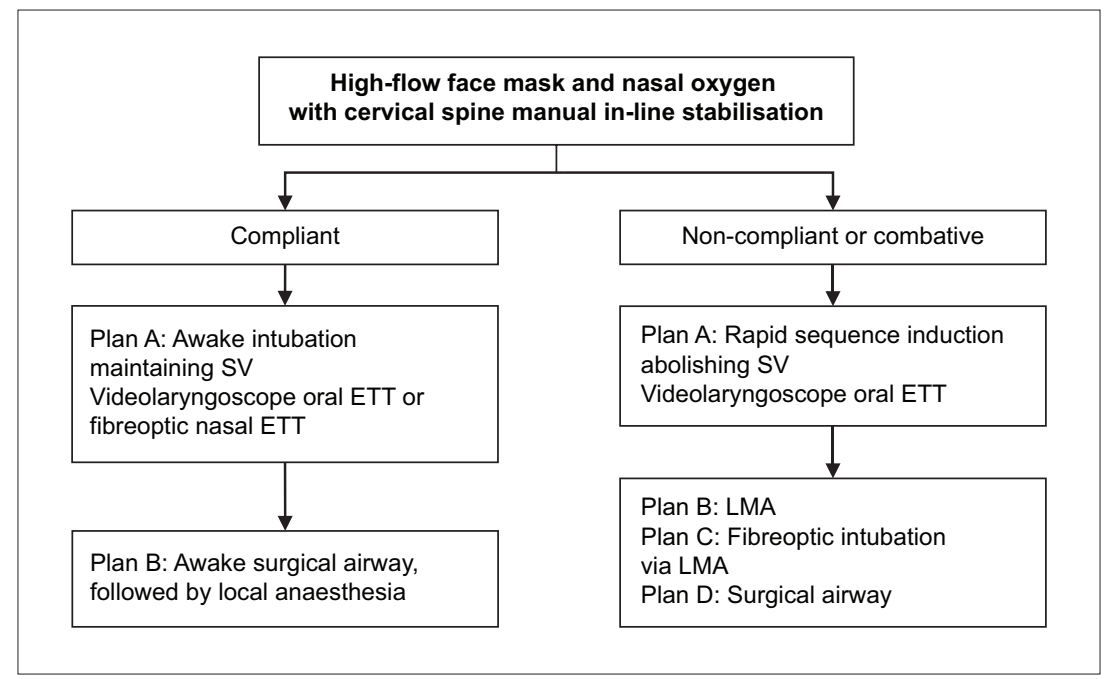

Fig. 2 Flowchart shows the decision-making process for upper airway management in inhalation injury. ETT: endotracheal tube; LMA: laryngeal mask airway; SV: spontaneous ventilation

Options for securing the airway include maintenance of spontaneous breathing with an awake intubation technique under upper airway local anaesthesia in compliant patients, or a rapid sequence induction (RSI) technique with general anaesthesia and abolishment of spontaneous breathing in non-compliant patients. If an RSI technique is chosen, it is important to note that suxamethonium is contraindicated after the first 24 hours following acute burns, in view of the risk of hyperkalaemia. ${ }^{(12)}$ This may be relevant in patients who present late to the A\&E, or are transferred from regional centres, several hours after sustaining their injury. Routine preparation for intubation typically includes dual oxygen administration with nasal and facial high-flow oxygen, as well as ensuring the availability of airway suctioning equipment and advanced airway equipment, including a videolaryngoscope and fibreoptic laryngoscope. Manual in-line stabilisation of the cervical spine is required if there is a history of trauma. A plan for rescue airway management and oxygenation in the event of a failed intubation should include availability of supraglottic airway devices, as well as surgical or percutaneous cricothyroidotomy techniques. ${ }^{(14-17)}$ In our case series, all patients were intubated uneventfully using an RSI technique. Patients 2, 3 and 4 were deemed to require prophylactic intubation in the A\&E in view of their altered mental status (Patients 2 and 4) or significant oedema on fibreoptic laryngoscopy (Patients 2 and 3). Patients 1 and 5 were considered stable for transfer to the EOT for endotracheal intubation, which is the case for the majority of our patients.

Patients with inhalation injury have a higher risk of failed intubation as well as a lower likelihood of successful rescue oxygenation and ventilation. In the event of failure or if there is any delay in securing the airway, we produced an aidememoire (Fig. 2) as a reminder to consider awake intubation if possible, with RSI being reserved for situations where an awake technique is not feasible (e.g. non-compliant patient or one with impending cardiorespiratory arrest). In our experience, awake videolaryngsocopic oral intubation is well tolerated by the majority of patients, who would also have recently undergone a fibreoptic laryngoscopy in the A\&E during initial triage. Effective upper airway local anaesthetic topicalisation is reliably achieved using a spray-as-you-go technique with a laryngotracheal mucosal atomisation device (MADgic ${ }^{\mathrm{TM}}$, Teleflex, Wayne, PA, USA). Local anaesthetic spray to the posterior third of the tongue, posterior pharyngeal wall, uvula and peritonsillar region is reliable for abolishing the gag reflex, which is essential for safe awake intubation. Supraglottic spray to the epiglottis, vallecula, aryepiglottic folds, arytenoids and vocal cords as well as subglottic local anaesthetic spray through the vocal cords, under video or fibreoptic laryngoscopic visualisation, avoids the need for transtracheal injections of local anaesthetic that are potentially dangerous in a patient with inhalation injury and upper airway oedema.

Fibreoptic bronchoscopy should only be performed after the airway is secured, ventilation is controlled and the patient's condition is stabilised. Unless there is any refractory hypoxaemia or lobar collapse, urgent surgery for burns or trauma should be performed prior to diagnostic fibreoptic bronchoscopy. Bronchoalveolar lavage and suctioning should be performed to clear obstructed airways and to obtain samples for microbiology.

In Endorf and Gamelli's ${ }^{(18)}$ retrospective chart review over three years, 80 patients were identified with inhalation injury, which required intubation and mechanical ventilation as well as fibreoptic bronchoscopy in the first 24 hours. The bronchoscopic abbreviated injury score (AIS) of the patients was found to correlate with mortality. The AIS is a grading system for inhalation injury that is scored according to the presence of carbonaceous deposits, bronchorrhoea, erythema, oedema, mucosal friability, sloughing and necrosis, bronchial obstruction, and endoluminal obliteration. The overall AIS is graded according to the worst feature present in any category. ${ }^{(3)}$ In our practice, however, we found that this may lead to differences in grading between different observers. Of particular note, there may be a difference between the severity and distribution of each component of the score, especially the degree of inflammation, degree of mucosal damage and carbonaceous deposits. This is illustrated in images 

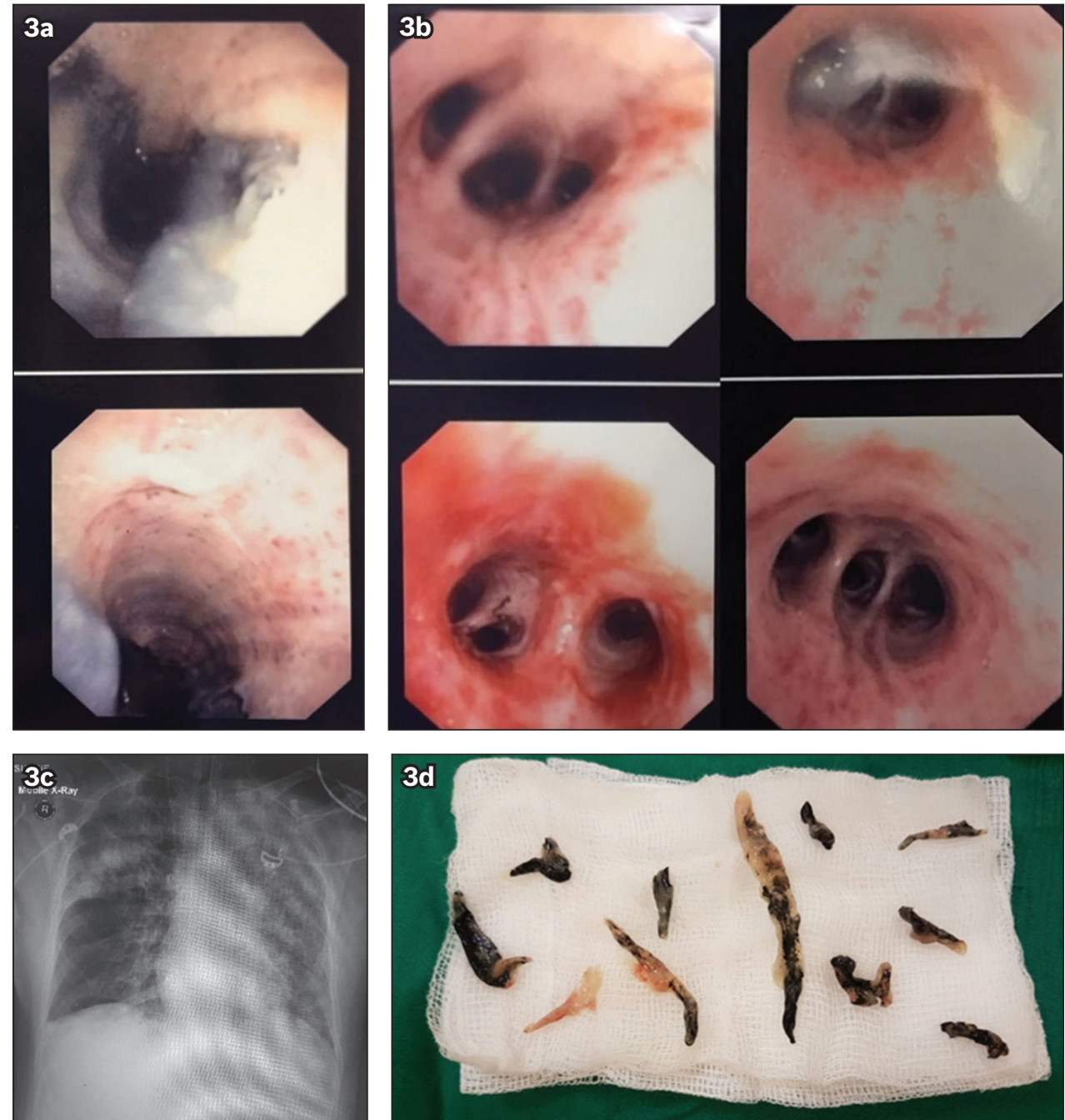

Fig. 3 Images of Patient 1 collected during his acute respiratory distress syndrome (ARDS) phase while in the BICU. Bronchoscopic images show (a) bronchorrhoea; and (b) bronchial mucosal erythema and bleeding. (c) Chest radiograph shows ARDS. (d) Photograph shows carbonaceous deposits extracted from bronchoalveolar lavage.

from Patient 1 (Fig. 3) showing a heterogeneous distribution of carbonaceous deposits, bronchorrhoea, bronchial mucosal erythema and mucosal bleeding (Figs. 3b-d). Therefore, we produced a simple modification, representing the individual components of the Endorff and Gamelli score in separate columns for clarity (Table III). The final grading is allocated according to the worst score of any component, in keeping with the recommendations of the original scoring system. We found that this approach has anecdotally improved interobserver concordance in scoring.

Lower airway fibreoptic bronchoscopic AIS is particularly useful in planning for early extubation following burns surgery in lower grades of injury (AIS 1-2) and predicting the need for more prolonged ventilation in higher grades of injury (AIS 3-4). AIS correlates with histologic findings of inhalation injury from bronchial biopsy, risk of ARDS, deteriorating oxygenation, higher $\mathrm{COHb}$ levels, prolonged ventilation, organ dysfunction and mortality. ${ }^{(19-23)}$ In more severe cases, the need for repeat bronchoscopy for sputum clearance, bronchoalveolar lavage for the diagnosis of pneumonia, and monitoring mucosal recovery can be predicted. (24-26) Virtual bronchoscopy, from three-dimensional reconstructions of thoracic computerised tomography (CT) images, has a high negative predictive value for excluding airway narrowing and oedema in an animal model of inhalation injury. ${ }^{(27)}$ In addition, virtual bronchoscopy was found to be reliable in confirming the diagnosis of inhalation injury in a small clinic study of ten patients. ${ }^{(28)}$ It might be possible to apply this to the initial triage of patients in A\&E, following upper airway fibreoptic laryngoscopy, to exclude overt airway obstruction with a high degree of reliability and minimise any delay in diagnosis. Virtual bronchoscopy may also play a role in patients in whom $\mathrm{CT}$ is being performed for other reasons, for example if there is coexisting trauma or suspicion of a pulmonary embolism. However, its limitations include the requirement for high-resolution CT image reconstruction and expertise in specialised image interpretation.

Hence, even though virtual bronchoscopy appears to be a promising diagnostic modality, further validation studies are required. In addition, it may not fully replace fibreoptic bronchoscopy, which has the advantage of allowing therapeutic suctioning of obstructing casts and the relief of life-threatening airway obstruction. Fibreoptic bronchoscopy has its own limitations, as it may prove challenging in hypoxic patients or if 
Table III. Modified Endorf and Gamelli classification separating components of the overall score for clarity.

\begin{tabular}{|c|c|c|c|c|}
\hline $\begin{array}{l}\text { Grade of } \\
\text { injury }\end{array}$ & Mucosal appearance & Carbonaceous deposits & Bronchorrhoea & Bronchial obstruction \\
\hline 0 (none) & No erythema & None & None & None \\
\hline 1 (mild) & $\begin{array}{l}\text { Minor or patchy areas of } \\
\text { erythema }\end{array}$ & $\begin{array}{l}\text { Minor deposits in proximal } \\
\text { or distal bronchi }\end{array}$ & None & None \\
\hline 2 (moderate) & Moderate erythema & Moderate & Moderate & $\begin{array}{l}\text { With or without } \\
\text { compromise of the bronch }\end{array}$ \\
\hline 3 (severe) & $\begin{array}{l}\text { Severe inflammation; friable } \\
\text { mucosa }\end{array}$ & Copious & Copious & Bronchial obstruction \\
\hline 4 (massive) & $\begin{array}{l}\text { Severe inflammation; mucosal } \\
\text { sloughing or necrosis }\end{array}$ & Copious & Copious & Endoluminal obliteration \\
\hline
\end{tabular}

Abbreviated injury score is graded according to the worst feature present.

an endotracheal tube with a relatively narrow diameter is present. In our practice, we have introduced guidelines (Box 2) based on the AIS criteria.

Another consideration is the use of nebulised heparin and acetylcysteine. Nebulised acetylcysteine has a potent mucolytic effect, but it is also a mild irritant, hence is traditionally administered together with bronchodilators to reduce the risk of bronchospasm. Bronchodilators may also be indicated in patients with severe inhalation injury or reactive airway disease (e.g. asthma or chronic obstructive pulmonary disease). Nebulised heparin is thought to be beneficial in inhalation injury by reducing fibrin cast formation and bronchial obstruction. Studies have demonstrated a reduction in ventilator days with no adverse bleeding events in inhalation injury patients treated with nebulised heparin in combination with acetylcysteine and bronchodilators. The optimal dose of nebulised heparin has been identified as 10,000 $U$ every four hours. ${ }^{(29-31)}$

In making a decision regarding extubation, an airway plan must be formulated to decide whether to keep the patient intubated or consider early extubation according to conventional clinical criteria, including upper airway patency, neurological status, muscular weakness, respiratory secretions and chest compliance. In cases of resolving upper airway swelling, it may be helpful to plan extubation according to the presence of a positive endotracheal tube cuff leak test. A short course of dexamethasone may help to reduce swelling and extubation, while leaving an airway exchange catheter in situ may confer an additional degree of safety. ${ }^{(32}$ Application of facial continuous positive airway pressure or noninvasive ventilation via a tight-fitting face mask may not be possible in patients with facial burns; however, high-flow humidified oxygen therapy via nasal cannulae following extubation is emerging as a suitable alternative in other areas of critical care. ${ }^{(33,34)}$ Specific factors to consider prior to extubation include the presence of: (a) upper airway thermal injury or swelling; (b) lower airway thermal injury or ARDS and whether further bronchoscopy is required to clear carbonaceous plugs; (c) bronchospasm; (d) pneumonia; (e) further surgery required; (f) ICU severity of illness and trends; (g) coexisting traumatic injuries or surgical/medical conditions; and (h) comorbidity, frailty and functional capacity.

Smailes et $\mathrm{al}^{(35)}$ retrospectively reviewed the incidence of extubation failure in a cohort of patients managed in a tertiary

\section{Box 2. Guidelines based on abbreviated injury score (AIS):}

\section{All patients}

- Lung-protective ventilation

- Standard intensive care management

- Intravenous fluids as per burns protocol, with caution to avoid positive fluid balance in view of the risk of worsening acute respiratory distress syndrome

AIS 1-2

- Mucolytics

- Chest physiotherapy

- Early extubation if:

- No significant upper airway swelling

- Successful ventilatory weaning and spontaneous breathing trial

- Low or improving sequential organ failure assessment score

- No further immediate surgical interventions planned

AIS 3-4

- Mucolytics

- Chest physiotherapy

- Repeat bronchoscopy with bronchoalveolar lavage for sputum clearance and diagnosis of pneumonia, and to monitor mucosal recovery prior to planning extubation

- In addition, consider virtual bronchoscopy to exclude overt airway obstruction if a thoracic computed tomography scan is performed for other indications (e.g. suspicion of pulmonary embolism)

BICU and requiring mechanical ventilation for longer than 24 hours. Of 60 patients planned to be extubated, 20 (33.3\%) failed this and required reintubation within 48 hours. There was no significant difference in severity of inhalation injury and burn size in patients who were successfully or unsuccessfully extubated. Patients who failed extubation had a longer overall duration of mechanical ventilation as well as longer intensive care and hospital lengths of stay. In 15 (75.0\%) out of 20 patients, impaired clearance of secretions was found to be a major risk factor for extubation failure.

In a separate study, Smailes et al ${ }^{(36)}$ determined that a weak cough as well as copious respiratory secretions were major aetiological factors in burns patients requiring reintubation following a successful spontaneous breathing trial. While tracheostomy may be beneficial in facilitating ventilatory weaning and pulmonary toilet, it is associated with a high incidence of 


\section{Accident and Emergency Department}

- Initial triage and resuscitation

- Upper airway nasolaryngoscopy and evaluation of upper airway

inhalation injury

- Limitation of intravenous fluids prior to intubation

Emergency operating theatre
- Definitive airway management
- Damage control surgery
- Fibreoptic bronchoscopy and evaluation of lower airway
inhalation injury

$\mid$

\section{Intensive care unit}

- Respiratory physiotherapy and mucolytics

- Repeat bronchoscopy for sputum clearance and diagnosis

of pneumonia

- Weaning ventilation

- Extubation planning

Fig. 4 Flowchart shows our recommended standardised workflow for patients with inhalation injury.

complications including a high incidence of subglottic stenosis in patients with moderate to severe inhalation injury. ${ }^{(37)}$ At present, there is no consensus on the indications and timing for tracheostomy in this group of patients, and decisions must be made after considering the balance of risks and benefits in the individual patient.

In conclusion, inhalation injury results in specific complications related to airway management. Based on our current case series and literature reviews, we propose the use of a standardised approach to management for this group of patients (Fig. 4). In this workflow, management is separated into three stages: A\&E, where nasoendoscopy is used to assess the upper airway; EOT, where fibreoptic bronchoscopy, evaluation of the lower airway and treatment for cutaneous burns are performed; and ICU, for monitoring and extubation planning.

\section{REFERENCES}

1. Carmont MR. The Advanced Trauma Life Support course: a history of its development and review of related literature. Postgrad Med J 2005; 81:87-91.

2. Dries DJ, Endorf FW. Inhalation injury: epidemiology, pathology, treatment strategies. Scand J Trauma Resusc Emerg Med 2013; 21:31.

3. Walker PF, Buehner MF, Wood LA, et al. Diagnosis and management of inhalation injury: an updated review. Crit Care 2015; 19:351.

4. Gill P, Martin RV. Smoke inhalation injury. Contin Educ Anaesth Crit Care Pain 2015; 15:143-8.

5. Wise B, Levine Z. Inhalation injury. Can Fam Physician 2015; 61:47-9

6. Madnani DD, Steele NP, de Vries E. Factors that predict the need for intubation in patients with smoke inhalation injury. Ear Nose Throat J 2006; 85:278-80.

7. Onishi S, Osuka A, Kuroki Y, Ueyama M. Indications of early intubation for patients with inhalation injury. Acute Med Surg 2017; 4:278-85.

8. Ching JA, Shah JL, Doran CJ, et al. The evaluation of physical exam findings in patients assessed for suspected burn inhalation injury. J Burn Care Res 2015; 36:197-202.

9. Muehlberger T, Kunar D, Munster A, Couch M. Efficacy of fiberoptic laryngoscopy in the diagnosis of inhalation injuries. Arch Otolaryngol Head Neck Surg 1998; 124:1003-7.

10. Goh SH, Tiah L, Lim HC, Ng EK. Disaster preparedness: experience from a smoke inhalation mass casualty incident. Eur J Emerg Med 2006; 13:330-4.

11. Ikonomidis C, Lang F, Radu A, Berger MM. Standardizing the diagnosis of inhalation injury using a descriptive score based on mucosal injury criteria. Burns 2012; 38:513-9.

12. Bishop S, Maguire S. Anaesthesia and intensive care for major burns. Contin Educ Anaesth Crit Care Pain 2012; 12:118-22.

13. Oscier C, Emerson B, Handy JM. New perspectives on airway management in acutely burned patients. Anaesthesia 2014; 69:105-10.

14. Hagberg CA, Gabel JC, Connis RT. Difficult Airway Society 2015 guidelines for the management of unanticipated difficult intubation in adults: not just another algorithm. Br J Anaesth 2015; 115:812-4.

15. Apfelbaum JL, Hagberg CA, Caplan RA, et al; American Society of Anesthesiologists Task Force on Management of the Difficult Airway. Practice guidelines for management of the difficult airway: an updated report by the American Society of Anesthesiologists Task Force on Management of the Difficult Airway. Anesthesiology 2013; 118:251-70.

16. Weingart SD, Levitan RM. Preoxygenation and prevention of desaturation during emergency airway management. Ann Emerg Med 2012; 59:165-75.e1.

17. Patel A, Nouraei SA. Transnasal Humidified Rapid-Insufflation Ventilatory Exchange (THRIVE): a physiological method of increasing apnoea time in patients with difficult airways. Anaesthesia 2015; 70:323-9.

18. Endorf FW, Gamelli RL. Inhalation injury, pulmonary perturbations, and fluid resuscitation. J Burn Care Res 2007; 28:80-3.

19. Masanes MJ, Legendre C, Lioret N, et al. Fiberoptic bronchoscopy for the early diagnosis of subglottal inhalation injury: comparative value in the assessment of prognosis. J Trauma 1994; 36:59-67.

20. Masanès MJ, Legendre C, Lioret N, Saizy R, Lebeau B. Using bronchoscopy and biopsy to diagnose early inhalation injury. Macroscopic and histologic findings. Chest 1995; 107:1365-9.

21. Mosier MJ, Pham TN, Park DR, et al. Predictive value of bronchoscopy in assessing the severity of inhalation injury. J Burn Care Res 2012; 33:65-73.

22. Spano S, Hanna S, Li Z, Wood D, Cartotto R. Does bronchoscopic evaluation of inhalation injury severity predict outcome? J Burn Care Res 2016; 37:1-11.

23. Marek K, Piotr W, Stanisław S, et al. Fibreoptic bronchoscopy in routine clinical practice in confirming the diagnosis and treatment of inhalation burns. Burns 2007; 33:554-60.

24. Bai $C$, Huang $H$, Yao X, et al. Application of flexible bronchoscopy in inhalation lung injury. Diagn Pathol 2013; 8:174.

25. Carr JA, Crowley N. Prophylactic sequential bronchoscopy after inhalation injury: results from a three-year prospective randomized trial. Eur J Trauma Emerg Surg 2013; 39:177-83.

26. Carr JA, Phillips BD, Bowling WM. The utility of bronchoscopy after inhalation injury complicated by pneumonia in burn patients: results from the National Burn Repository. J Burn Care Res 2009; 30:967-74.

27. Kwon HP, Zanders TB, Regn DD, et al. Comparison of virtual bronchoscopy to fiber-optic bronchoscopy for assessment of inhalation injury severity. Burns 2014; 40:1308-15

28. Gore MA, Joshi AR, Nagarajan G, et al. Virtual bronchoscopy for diagnosis of inhalation injury in burnt patients. Burns 2004; 30:165-8.

29. Elsharnouby NM, Eid HE, Abou Elezz NF, Aboelatta YA. Heparin/Nacetylcysteine: an adjuvant in the management of burn inhalation injury: a study of different doses. J Crit Care 2014; 29:182.e1-4.

30. Otremba S, Faris J, Zimmerman LH, Barbat S, White M. Inhaled heparin in critically ill patients with smoke inhalation injury. Crit Care Med 2013; 41:A231-2.

31. Mclntire AM, Harris SA, Whitten JA, et al. Outcomes following the use of nebulized heparin for inhalation injury (HIHI study). J Burn Care Res 2017; 38:45-52.

32. Greenhalgh DG, Warner P. Potential studies in extubation adjuncts after inhalation injury. J Burn Care Res 2009; 30:199-200.

33. Hernández G, Vaquero C, Colinas L, et al. Effect of postextubation high-flow nasal cannula vs noninvasive ventilation on reintubation and postextubation respiratory failure in high-risk patients: a randomized clinical trial. JAMA 2016; 316:1565-74

34. Stéphan F, Barrucand B, Petit P, et al; BiPOP Study Group. High-flow nasal oxygen vs noninvasive positive airway pressure in hypoxemic patients after cardiothoracic surgery: a randomized clinical trial. JAMA 2015; 313:2331-9.

35. Smailes ST, Martin RV, McVicar AJ. The incidence and outcome of extubation failure in burn intensive care patients. J Burn Care Res 2009; 30:386-92.

36. Smailes ST, McVicar AJ, Martin R. Cough strength, secretions and extubation outcome in burn patients who have passed a spontaneous breathing trial. Burns 2013; 39:236-42

37. Qing Y, Cen Y, Liu XX, Xu XW, Wang HS. [Analysis of extubation time and late complications after early tracheotomy in patients with inhalation injury]. Zhonghua Shao Shang Za Zhi 2011; 27:131-4. Chinese. 